1 Universidade de São Paulo (USP), Escola de Enfermagem, Departamento de Enfermagem Materno Infantil e Psiquiátrica - São Paulo (SP), Brasil.

vargas@usp.br

2 Universidade de São Paulo (USP), Escola de Enfermagem - São Paulo (SP), Brasil. janainasoares@usp.br 3 Universidade de São Paulo (USP), Escola de Enfermagem - São Paulo (SP), Brasil.

egleonr@usp.br

4 Universidade de São Paulo (USP), Escola de Enfermagem,

Departamento de Enfermagem Materno Infantil e Psiquiátrica - São Paulo (SP), Brasil.

caroline.figueira.pereira@ usp.br

${ }^{5}$ Universidade de São Paulo (USP), Escola de Enfermagem, Departamento de Enfermagem Materno Infantil e Psiquiátrica - São Paulo (SP), Brasil.

talitadp@usp.br

\section{O primeiro contato com as drogas: análise do prontuário de mulheres atendidas em um serviço especializado}

\author{
The first contact with drugs: analysis of medical records of women \\ cared in a specialized service
}

Divane de Vargas ${ }^{\mathbf{1}}$, Janaina Soares $\mathbf{2}$, Erika Leon $^{\mathbf{3}}$, Caroline Figueira Pereira ${ }^{\mathbf{4}}$, Talita Dutra Ponce $\mathbf{5}$
RESUMO O estudo objetivou caracterizar e compreender o contexto em que se dá o primeiro contato de mulheres com problemas relacionados ao uso de substâncias psicoativas que procuraram atendimento em um serviço especializado em álcool e outras drogas. Trata-se de um estudo exploratório, descritivo de abordagem quanti-qualitativa. Utilizaram-se informações contidas em 412 prontuários de mulheres atendidas num serviço especializado em álcool e outras drogas. Realizou-se uma análise exploratória dos dados quantitativos, e os dados qualitativos foram agrupados e analisados segundo a análise de conteúdo, originando cinco temáticas. Resultados: A idade média de primeiro contato com as drogas foi 16 anos e as substâncias mais usadas foram álcool, tabaco e maconha. Concluiu-se que, na adolescência, as mulheres ficam mais vulneráveis ao primeiro contato com substâncias psicoativas e que o contexto social e familiar exerce influência na iniciação do uso de drogas.

PALAVRAS-CHAVE Mulheres; Alcoolismo; Transtornos relacionados ao uso de substâncias.

\begin{abstract}
This study aimed to characterize and understand the circumstance under wich women carrying problems related to psychoactive substance use had their first contact with a specialized service in alcohol and other drugs. Exploratory and descriptive study of quantitative and qualitative approach. We retrieved of the information contained in medical records of 412 women cared in a service specializing in alcohol and other drugs. We held a quantitative data exploratory analysis and grouped qualitative data, wich were analyzed as for their content analysis, resulting in five themes. Results: the average age of women first contact with the drugs was 16 years, being alcohol, tobacco and marijuana the most commonly used substances. We concluded that, during adolescence, women are more vulnerable to the first contact with psychoactive substances, and that social and family contexts exert influence on the initiation of drug use.
\end{abstract}

KEYWORDS Women; Alcoholism; Substance-related disorders. 


\section{Introdução}

Atualmente, o uso de Substâncias Psicoativas (SPAs) pela população feminina tem aumentando, pois, quando comparados os dois últimos levantamentos domiciliares realizados no Brasil, observou-se que, entre os anos 2001 e 2005, o uso de álcool na vida por mulheres aumentou de $60,6 \%$ para $68,3 \%$, assim como a dependência de álcool, cresceu de $5,7 \%$ para $6,9 \%$ (CARLINI ET AL., 2006). No que se refere às drogas estimulantes, benzodiazepínicos, analgésicos (opiáceos) e orexígenos, as mulheres apresentaram prevalência de uso durante a vida aproximadamente duas a três vezes maior do que os homens. O uso de SPAs pelas mulheres pode levar ao desenvolvimento de problemas fisiológicos e psicossociais mais rapidamente do que na população masculina. Isso pode ocorrer devido às próprias características fisiológicas da mulher, pois, ao consumir álcool, a mulher tem mais facilidade para desenvolver a dependência, uma vez que possui maior proporção de tecido adiposo no corpo, que retém o álcool por maior tempo no organismo, e pela menor concentração gástrica da enzima desidrogenase alcoólica, fundamental na decomposição do álcool (BRASIL, 2013).

O uso de SPAs pela população feminina é pouco aceito socialmente, levando-as a apresentar preferência pelo uso de substâncias lícitas, como medicamentos, álcool e tabaco. Essas preferências nem sempre despertam a atenção dos pesquisadores, que priorizam os estudos envolvendo o uso das substâncias ilícitas (VASCONCELOS, 2006).

Em relação à saúde da mulher, os estudos, em sua maioria, estiveram voltados para questões reprodutivas, maternas, e a desigualdades entre homens e mulheres, fatores que exerceram impacto significativo em suas condições de saúde (BRASIL, 2004). Entretanto, estudo realizado no Brasil (GALERA; ROLDÁN; O'BRIEN, 2005) demonstrou que o uso de SPAs entre as mulheres inicia-se geralmente na adolescência e tem como maior fator motivador a pressão social e a violência familiar. Outros autores apontam que o uso de SPAs por mulheres pode estar relacionado à grande mudança no perfil da mulher contemporânea, o que acarretou atividades em excesso, dificuldade em lidar com os problemas, principalmente problemas familiares, ansiedade e estresse. Essas situações podem ser determinantes na realização de suas escolhas e no uso prejudicial de SPAs (MALBERGIER ET AL., 2012; BARCINSKI, 2009).

Os ambientes em que as mulheres e os homens fazem uso de SPAs são diferentes; geralmente as mulheres fazem uso em casa, sozinhas ou apenas com amigas, enquanto os homens consomem em bares, ruas e festas. Essa especificidade das mulheres tem sido tema de interesse dos pesquisadores que apontam a importância de se investigar esse fenômeno, podendo, então, pensar em ações de saúde mais específicas para essa população, buscando resultados mais efetivos (BARCINSKI, 2009).

Considerando-se o aumento do consumo de SPAs em mulheres, assim como de morbidades consequentes do uso prejudicial e do número de mulheres dependentes dessas substâncias que procuram tratamento nos serviços especializados, é esperado que haja estudos publicados sobre a temática, porém ainda são escassos na literatura estudos relacionados aos problemas decorrentes do uso de SPAs entre as mulheres, pois as investigações nessa área são, em sua maioria, focadas na população masculina (COOK, 2004). Isso ocorre, segundo Rennó Jr. et al. (2005), pelo fato de as mulheres terem permanecido, durante algumas décadas, pouco representadas nas pesquisas, em especial por não procurarem tratamento. Nesse contexto, julgou-se necessário realizar um estudo para caracterizar o primeiro contato de mulheres que procuraram tratamento para o uso de SPAs em um serviço especializado da cidade de São Paulo. Essa caracterização poderá fornecer subsídio para futuras estratégias de 
ações em saúde que possam levar em consideração as especificidades encontradas.

Os objetivos da pesquisa foram: Caracterizar o primeiro contato de mulheres com problemas relacionados ao uso de SPAs que procuraram atendimento em um serviço especializado em álcool e outras drogas; Compreender o contexto do primeiro uso de SPAs por mulheres com problemas relacionados ao uso de SPAs que buscaram atendimento em um centro de tratamento para o álcool e outras drogas.

\section{Método}

Estudo do tipo exploratório, descritivo, com abordagem quantitativa e qualitativa, secundário a um projeto maior que visou a identificar o perfil de mulheres que procuram tratamento para dependência química em um serviço especializado do estado de São Paulo. Este estudo configura-se como descritivo porque permite descrever o conhecimento sobre a realidade histórica e social do contexto em que se situa o objeto e possibilita explorar dimensões de um objeto, na forma como se apresenta, para ampliar o entendimento de sua característica (RODRIGUES; SANTOS; SPRICCIGO, 2012).

A coleta dos dados se deu por meio de busca nos prontuários de todas as mulheres que foram atendidas pela primeira vez em um serviço especializado em álcool e outras drogas da cidade de São Paulo, no período compreendido entre janeiro de 2001 a dezembro de 2010. Esse período foi escolhido por caracterizar-se como a primeira década de funcionamento do serviço como referência para o tratamento de transtornos relacionados ao uso de SPAs no município. Os dados foram coletados entre janeiro e março de 2011. Os critérios de exclusão foram prontuários de mulheres que apresentavam comorbidades psiquiátricas, que procuraram o serviço para o tratamento da obesidade ou tratamento exclusivo para o tabagismo.
Utilizaram-se as informações contidas em 412 prontuários de mulheres atendidas nesse serviço especializado, sendo que os dados quantitativos coletados referentes à idade, tipo de droga de primeiro contato e contexto de utilização da droga foram armazenados no programa SPSS (Statistical Package for the Social Sciences), utilizado para a análise exploratória dos dados.

Os dados qualitativos relacionados ao primeiro contato com as drogas foram obtidos de respostas à questão aberta previamente realizada e registrada no prontuário pelos profissionais de saúde presentes no atendimento inicial: 'Como foi o primeiro contato com as drogas'. Desses prontuários, somente 218 foram escolhidos por terem resposta para essa pergunta. Para análise dessa informação, utilizou-se a técnica de análise de conteúdo de Bardin (2002) com o intuito de estabelecer correspondências entre as estruturas semânticas ou linguísticas (que estão na superfície do texto) e as estruturas psicológicas ou sociológicas (que determinam as características encontradas nos textos). Estas últimas se relacionam com a noção do contexto da mensagem, que possibilita o aprofundamento do significado, indo além das aparências. Essa técnica baseia-se em operações de desmembramento do texto em unidade, ou seja, esta etapa visa a descobrir os diferentes núcleos de sentido que constituem a comunicação e, posteriormente, realizar o seu reagrupamento em classes ou categorias (BARDIN, 2002).

A repetição e o reagrupamento dos dados gerou a categoria de interesse 'o primeiro contato com as drogas', da qual se originaram cinco temáticas para análise: I - Primeiro contato por influência de amigos e em momentos de descontração, II - Contexto familiar e de relacionamentos, III - Contexto escolar, IV - Contexto das ruas após momentos de estresse e perdas e V - Contexto do trabalho.

Esse projeto foi aprovado pelo Comitê de Ética em Pesquisa com seres humanos da 
Escola de Enfermagem da Universidade de São Paulo pelo Processo de $n^{0} 865 / 2009$.

\section{Resultados}

Os resultados demonstraram que, do número total de prontuários $(\mathrm{n}=412), 335$ $(81,3 \%)$ informavam a idade de primeiro contato com as drogas, sendo 16 a média de idade encontrada. Com relação à primeira droga utilizada, 315 prontuários $(76,5 \%)$ tinham essa informação. Desses prontuários, 273 mulheres $(86,45 \%)$ informaram ter tido o primeiro contato com uma única droga, enquanto 42 mulheres (13,3\%) informaram que o primeiro contato ocorreu com múltiplas drogas na mesma ocasião. A substância mais experimentada foi o álcool, seguido do tabaco e da maconha (gráfico 1). Dos 412 prontuários analisados, $218(53 \%)$ continham resposta à pergunta aberta 'Como foi o primeiro contato com as drogas?' (tabela 1).

Gráfico 1. Distribuição das frequências relativas e absolutas, conforme a primeira droga de uso, São Paulo, 2013

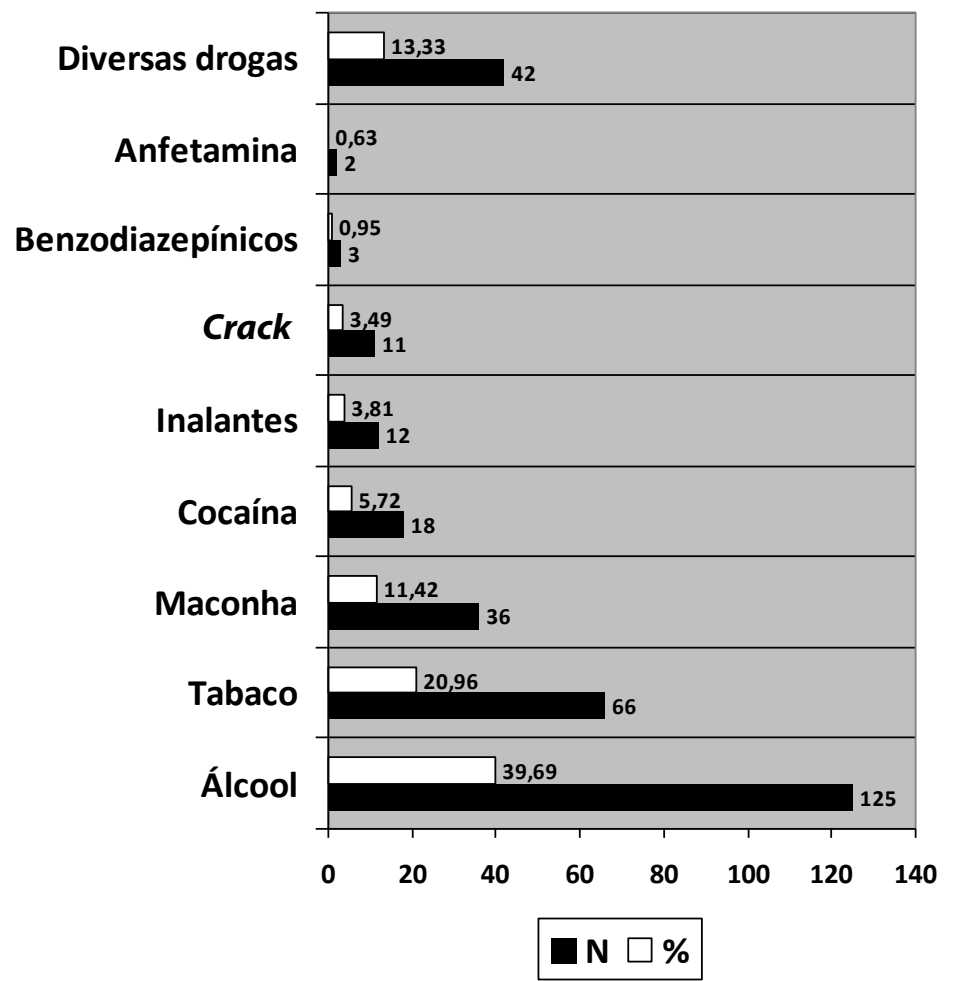




\begin{tabular}{lll}
\hline Tabela 1. Distribuição das frequências relativas e absolutas, conforme o primeiro contato com as drogas. São Paulo, 2013 \\
\hline Variáveis & Frequência & $\%$ \\
\hline Com amigos & 64 & 29,36 \\
Em festas, bares, baladas, etc. & 35 & 16,05 \\
Com familiares & 32 & 14,68 \\
Em casa & 23 & 10,55 \\
Na rua & 16 & 7,34 \\
Na escola & 9 & 4,13 \\
Após perdas ou separações & 7 & 3,21 \\
Com o namorado & 6 & 2,75 \\
No trabalho & 6 & 2,75 \\
Outros & 20 & 9,18 \\
\hline Total & 218 & 100 \\
\hline
\end{tabular}

Nota: Elaboração própria

\section{Análise categorial - temática}

As respostas encontradas nos prontuários referentes ao primeiro contato com as drogas, foram agrupadas em uma categoria simples de análise: $\mathrm{O}$ primeiro contato com as drogas, que originou cinco temáticas relacionadas aos grupos, locais e motivos, relatados nos prontuários como circunstâncias que levaram ao primeiro contato com as drogas.

\section{I - O PRIMEIRO CONTATO POR INFLUÊNCIA DE AMIGOS E EM MOMENTOS DE DESCONTRAC̣ÃO}

Nas respostas dadas à questão: 'Como foi seu primeiro contato com as drogas?', observou-se que o primeiro contato ocorreu junto com amigos, como aparece na fala: "No banheiro da escola com meus amigos" (P1). Conforme algumas respostas, esse contato com as drogas por meio dos amigos esteve relacionado a um sentimento de aceitação no grupo: "Bebia com meus amigos para me sentir "legal”" (P250). Assim como relacionado a confraternizações: "Com meus amigos, para comemorar" (P300); "Em uma festa com meus amigos" (P4); "Nos finais de semana, em churrascos com os meus amigos"
(P5). "E também por prazer: Comecei tomando cerveja em busca de prazer com amigos" (P16).

A análise das respostas mostrou, ainda, que o primeiro uso ocorreu no contexto de festas, bares, baladas etc., conforme indicam alguns relatos: "No baile funk" (P70); "No bar" (P8); "Em festas" (P95); "Em festas, bares e baladas" (P10); "Foi em uma danceteria" (P110). Ou, ainda, fizeram o primeiro uso como uma forma de encorajar o enfrentamento de situações estressantes, representadas pela facilidade de socialização trazida: "Eu usava para ir para festas" (P12).

\section{II - O PRIMEIRO CONTATO NO CONTEXTO FAMILIAR E DE RELACIONAMENTOS}

No que refere às relações familiares e relacionamentos afetivos, as respostas encontradas nos prontuários sugerem que o primeiro contato com as drogas se deu sob a influência de algum familiar, como observado nas seguintes respostas:

"Com meu pai" (P3); "Com meu primo em Curitiba" (P149); "Foi minha cunhada que me ofereceu" (P25); "Minhas tias me ofereciam a bebida" (P16). Ou em circunstâncias 
que envolviam familiares: "Foi em um churrasco em casa, com minha família. Fiquei alcoolizada e fumei crack sem saber o que era" (P77); "Meu tio esqueceu um cigarro ao meu alcance" (P83); "Comecei por curiosidade. Enrolei um cigarro com o fumo de corda que minha mãe usava" (P92); "Comecei socialmente no boteco do meu pai. Roubava do meu pai” (P20).

As respostas também mostram que as mulheres fazem o primeiro uso no ambiente domiciliar, geralmente às escondidas: "Eu tomava álcool em casa (P115); Bebi escondido em casa, mas não gostei porque passei mal" (P222); "No portão da minha casa" (P230); "Fumei sozinha escondida em casa" (P214).

Também aparecem respostas do primeiro uso no contexto dos relacionamentos afetivos, como o namorado, às vezes, pela facilitação do parceiro em adquirir a droga ou em companhia para o uso, como se pode observar nos seguintes registros: "Meu ex-namorado comprava bebida pra nós e depois costumava me agredir" (P401); "Através de um ex-namorado" (P412); "Usei a primeira vez com meu namorado" (P402); "Quando fugi de casa com um namorado de 19 anos" (P325). "Ou, ainda, por influência de terceiros: Um dos homens que me violentaram me deu drogas" (P311).

\section{III - O PRIMEIRO CONTATO NO CONTEXTO ESCOLAR}

O ambiente escolar também aparece nas respostas das mulheres como cenário do primeiro contato com as drogas, conforme ilustram os relatos: "Foi no banheiro da escola" (P132); "Na escola" (P130); "Na porta da escola" (P41); "Com meu professor, orientador de mestrado" (P58).

\section{IV - O PRIMEIRO CONTATO NO CONTEXTO DAS RUAS APÓS MOMENTOS DE ESTRESSE E PERDAS}

Algumas mulheres também relataram ter feito contato com as drogas na rua: "Na Praça da Sé” (P23); "Peguei uma bituca do chão na rua e experimentei por curiosidade" (P122). Alguns prontuários trazem registros do primeiro consumo na rua relacionando-o a eventos estressores, como ter sido expulsa de casa ou sofrido algum tipo de violência: "Fiquei grávida e minha mãe me expulsou de casa. Fiquei triste e comecei a beber na rua" (P86); "Fui aliciada aos 7 anos, não aguentei mais e fui embora de casa. Na rua, experimentei drogas" (P86).

Ainda aparecem nos prontuários o primeiro contato associado a situações de estresse ou perdas, conforme ilustram as respostas que seguem: "Foi após a separação dos meus pais; Após a separação do meu marido; Após a separação do meu primeiro marido" (P88); "Comecei a beber após o divórcio; Comecei o uso socialmente, mas se intensificou após meu divórcio" (P25); "Quando meu filho foi tirado de mim pelo pai dele" (P24); "Peguei meu namorado com uma garota de programa” (P398).

\section{V - O PRIMEIRO CONTATO NO CONTEXTO DO TRABALHO}

O ambiente de trabalho exerceu influência no primeiro contato das mulheres com as drogas, merecendo destaque as atividades relacionadas com o trabalho sexual ou com o tráfico de drogas: "Era profissional do sexo e comecei a beber" (P317); "Quando iniciei a atividade de profissional do sexo" (P173); "Eu arrumei um trabalho para vender drogas; foi no tráfico" (P83). 
Observaram-se também, relatos de primeiro contato com as drogas por meio dos colegas do trabalho: "Foi após começar a trabalhar na empresa $X$; todos os colegas bebiam" (P121); "Quando trabalhava em casa de família como doméstica P216; Eu trabalhava como doméstica e meu patrão me dava bebidas alcoólicas antes das refeições” (P52).

\section{Discussão}

A análise dos resultados permitiu observar que o primeiro contato com as drogas ocorreu no período da puberdade/adolescência. Esses resultados corroboram os estudos prévios que demonstraram a precocidade da primeira experimentação de SPAs pelas mulheres, entre 10 e 12 anos (GALDURÓZ ET AL., 2004) e entre 13 e 14 anos (MALTA ET AL., 2011). Também revelam que é no período da adolescência que a população, independentemente do sexo, fica mais vulnerável ao primeiro contato com SPAs. Uma vez que as transformações sofridas nesse período vêm, em geral, permeadas de dúvidas, instabilidade emocional e crise de identidade. Além desse fato, pode-se dizer que, por muitas vezes, a SPA é utilizada pelo adolescente como um meio de amenizar os problemas, aliviando as questões que poderiam ser enfrentadas de outras maneiras. Diante do exposto, nota-se a necessidade de trabalhar a prevenção do uso precoce de drogas na adolescência (CANAVEZ; ALVES; CANAVEZ, 2010).

Em relação à primeira SPA experimentada pelas respondentes, observou-se que as drogas lícitas (álcool e tabaco) ficaram respectivamente em primeiro e segundo lugar, seguidas da maconha, em terceiro lugar. Em estudo (BERNARDY; OLIVEIRA, 2010) realizado com jovens de 12 a 18 anos de idade, institucionalizados para tratamento de uso de SPAs, observou-se que a droga utilizada inicialmente pela maior parte dos adolescentes estudados foi a maconha, consumida diariamente, e gradativamente substituída pelo crack. Esse estudo revelou ainda que, dentre as drogas ilícitas, a maconha é a mais frequentemente escolhida pelos adolescentes, independentemente do sexo.

Outro fator importante na escolha das SPAs são os aspectos socioeconômicos: grande parte dos adolescentes não trabalha e não tem renda própria, levando essa população a optar por drogas de baixo custo, como o álcool e a maconha (Jesus et AL., 2011). Porém, independentemente do custo, o uso de drogas lícitas aumenta significativamente a associação com as ilícitas, sendo que a escala de progressão das SPAs é ordenada em um crescente de iniciação em que primeiramente vem o tabaco ou o álcool, seguido por outras substâncias ilícitas. Assim, a experimentação flui gradativa a partir de SPAs lícitas em direção às ilícitas (IGLESIAS ET AL., 2007).

A análise qualitativa relacionada ao primeiro contato das mulheres com as drogas permitiu compreender que o primeiro contato ocorreu juntamente com os amigos, e que essa experiência com o grupo de pares, na maioria das vezes, aconteceu em festas, bares e baladas ou até mesmo dentro da escola. Resultado semelhante foi encontrado em outro estudo (PRATTA; SANTOS, 2006), que revelou os grupos de pares e a família como os dois principais responsáveis pela iniciação dos adolescentes no uso de SPAs. De acordo com Canavez, Alvez, Canavez (2010), a experimentação acontece pelo fato de $o$ adolescente ter amigos que já usam drogas, gerando uma pressão do grupo na direção do uso.

Nos resultados deste estudo, pode-se observar que os familiares também foram citados por mais de $10 \%$ da população estudada, ocupando o terceiro lugar na lista das principais circunstâncias que levam ao primeiro contato com SPAs. A influência da família e dos amigos constitui-se como um dos principais responsáveis pela introdução dos adolescentes ao uso de SPAs. Isso, porque a família, pessoas próximas ou o grupo de amigos servem como modelo de comportamento, sendo que os últimos também exercem pressão social que, particularmente, influencia os adolescentes (DIETZ ET AL., 2011). Estudos demonstram que a 
dependência de drogas entre meninas está diretamente associada à falta de controle dos pais. Enquanto as meninas precisam de maior apoio da família como fator de proteção à dependência de drogas, os meninos necessitam de maior apoio de seus pares, sugerindo que as meninas respondem mais ao ambiente familiar (GRIFFIN ET AL., 2000).

Parcela significativa das respostas obtidas nos prontuários mostrou que o primeiro contato com as drogas aconteceu dentro da própria casa, escondido ou até mesmo junto com familiares. Estar em um ambiente onde o uso de SPAs é considerado comum leva a adolescente a consumir tais substâncias (CANAVEZ; AlVES; CANAVEZ, 2010). Assim, os dados desse estudo sugerem que a presença de um familiar usuário de SPAs pode aumentar a probabilidade de contato precoce das mulheres com as drogas.

Os resultados mostraram que em muitos casos o primeiro contato das mulheres com SPAs deu-se na rua. Estudos prévios (D'AMIC; MC CARTHY, 2006) associam o fato de pessoas em situação de rua ter maior uso SPAs devido à disponibilidade ou facilidade de acesso, propiciado pela inexistência de limites ou regras estabelecidas. Além disso, o fato de muitas mulheres estarem em situação de rua pode ter relação com a falta de opções de trabalho, baixa renda, dentre outros, o que proporciona maior vulnerabilidade para o envolvimento com o tráfico, e, assim, maior contato com as SPAs e seu uso (BARCINSKI, 2009), o que também foi demonstrado no mesmo estudo (BARCINSKI, 2009), onde o uso de substâncias também aconteceu após as mulheres começarem a trabalhar com o tráfico de drogas. Além disso, as situações às quais as pessoas que vivem na rua estão expostas, como fome, frio, violência, enfrentamento das adversidades em geral são fatores que predispõem ao uso de drogas.

Este estudo mostrou que o início do uso de SPAs pode estar relacionado com as perdas, separações e decepções decorrentes de relacionamentos afetivos e românticos. Isso sugere que os relacionamentos afetivos e românticos das adolescentes e as situações de crise podem constituir-se como fatores influentes para iniciação precoce do uso de SPAs. Ainda que mereçam atenção, esses não foram os motivos mais citados, o que sugere que não são os principais determinantes. Pratta e Santos (2006) sugeriram a 'curiosidade' como a principal razão dos adolescentes para o primeiro uso de drogas associados a contextos facilitadores, como amigos que já fazem uso de SPAs, fuga da realidade, dentre outros, os quais também foram encontrados neste estudo.

As limitações deste estudo compreendem a coleta dos prontuários, pois foi realizada em um único serviço especializado da cidade de São Paulo, e as características da população estudada, já que constituídas, em sua maioria, por usuárias moradoras de rua, sem apoio familiar e social, o que pode ter influenciado os resultados. Apesar das limitações apresentadas, este estudo é relevante, pois apresenta resultados relacionados a uma população pouco estudada no Brasil, não apenas quantitativamente como também na compreensão do contexto do primeiro contato com as SPAs por mulheres.

\section{Conclusão}

O estudo mostrou que a adolescência caracteriza-se por um período de suscetibilidade em relação ao primeiro contato com drogas e revelou, ainda, a grande influência do contexto social e familiar na iniciação do uso de SPAs. O primeiro contato se dá predominantemente com as drogas consideradas lícitas, as quais favorecem o uso das substâncias consideradas ilícitas. Os resultados revelam a necessidade de se iniciar o trabalho de prevenção ao uso precoce de SPAs entre as adolescentes, principalmente aquelas de caráter lícito. Tal decisão possibilita a reflexão acerca do emprego de estratégias de intervenção em saúde no âmbito domiciliar, uma vez que os resultados sugerem que o uso 
de SPAs tem início no meio familiar e ocorre de maneira reservada quando comparada à população masculina. Novos trabalhos devem ser realizados com intuito de identificar fatores capazes de retardar o primeiro contato das adolescentes com as SPAs.

Os achados também podem contribuir para a construção de estratégias futuras de prevenção e tratamento direcionadas a essa população. A construção dessas estratégias deve seguir as diretrizes da política nacional

\section{Referências}

BARCINSKI, M. Centralidade de gênero no processo de construção da identidade de mulheres envolvidas na rede do tráfico de drogas. Ciência e Saúde Coletiva, Rio de Janeiro, v. 14, n. 4, p. 1843-1853, 2009.

BARDIN, L. Análise de conteúdo. Lisboa: Edições 70, 2002.

BERNARDY, C. C. F.; OLIVEIRA, M. L. F. O papel das relações familiares na iniciação ao uso de drogas de abuso por jovens institucionalizados. Revista da Escola de Enfermagem da USP, São Paulo, v. 44, n. 1, p. 11-17, 2010.

BRASIL. Ministério da Justiça. Tratamento/ Populações específicas/ Mulheres. Disponível em: $<$ http://www.obid.senad.gov.br/portais/OBID/conteudo/index.php?id_conteudo $=11423 \&$ rastro $=$ TRATAME NTO\%2FPopula\%C3\%A7\%C3\%B5es+espec\%C3\%ADfi cas/Mulheres>. Acesso em: 11 jul. 2013.

. Ministério da Saúde. Secretaria de Atenção à Saúde. Departamento de Ações Programáticas Estratégicas. Política Nacional de Atenção Integral à Saúde da Mulher: princípios e diretrizes. Brasília, DF: Ministério da Saúde, 2004. Disponível em: <http://conselho.saude.gov.br/ultimas_noticias/2007/politica_mulher.pdf>. Acesso em: 27 jun. 2013.

CAMPOS, E. A.; REIS, J. G. Representations on the use of alcohol among women undergoing treatment at a reference center in the city of São Paulo, Brazil. Interface (Botucatu), Botucatu, v. 14, n. 34, p. 539-50, jul./set. 2010. de atenção integral à saúde da mulher, que preconiza a dinâmica inclusiva da gestão pública em todos os níveis assistenciais, de forma a atender às demandas emergentes e antigas da saúde da mulher. Dentre estas, as ações de redução da vulnerabilidade na adolescência não devem estar voltadas apenas para a redução de agravos à saúde sexual e reprodutiva da mulher (BRASIL, 2004), mas também a outros aspectos da vida dessas adolescentes, dentre eles o uso precoce de SPAs.
CANAVEZ, M. F.; ALVES, A. R.; CANAVEZ, L. S. Fatores predisponentes para o uso precoce de drogas por adolescentes. Cadernos UniFOA, Volta Redonda, n. 4, p. 57-63, 2010.

CARLINI, E. A. et al. II Levantamento domiciliar sobre uso de drogas psicotrópicas no Brasil - 2005. Principais resultados - Estudo comparativo Brasil - 2001 e 2005. São Paulo: Centro Brasileiro de Informações sobre Drogas Psicotrópicas. Universidade Federal de São Paulo, 2006. p. 303-319.

COOK, L. J. Educating women about the hidden dangers of alcohol. Journal Psychosocial Nurses Mental Health Service, Philadelphia, v. 42, n. 6, p. 24-31, jun. 2004.

D'AMICO, E. J.; MC CARTHY, D. M. Escalation and initiation of younger adolescents substance use: The impact of perceived peer use. Journal adolescent Health, Philadelphia, v. 39, n. 4, p. 481-487, 2006.

DIETZ, G. et al. As relações interpessoais e o consumo de drogas por adolescentes. SMAD, Revista Eletrônica Saúde Mental Álcool Drogas, Ribeirão Preto, v. 7, n. 2, p. 85-91, maio/ago. 2011.

GALDURÓZ, J. C. F. et al. V levantamento nacional sobre o consumo de drogas psicotrópicas entre estudantes de ensino fundamental e médio da rede pública de ensino nas 27 capitais brasileiras. São Paulo: Centro Brasileiro de informações sobre Drogas Psicotrópicas, 2004. 
GALERA, S. A. F.; ROLDÁN, M. C. B.; O'BRIEN, B. Mulheres vivendo no contexto de drogas (e violência) - papel maternal. Revista Latino-americana de Enfermagem, Ribeirão Preto, v. 13, número especial, nov./dez. 2005.

GRIFFIN, K. W. et al. Parenting practices as predictors of substance abuse. Psychology Addictive Behaviors, Washington, DC, n. 14, p. 174-84, 2000.

IGLESIAS, V. et al. Early tobacco and alcohol consumption as modifying risk factors on marijuana use. Revista de Saúde Pública, São Paulo, v. 41, p. 4, 2007.

JESUS, F. B. et al. Vulnerabilidade na Adolescência: uma Experiência e Expressão do Adolescente. Revista Gaúcha de Enfermagem, Porto Alegre, v. 32, n. 2, p. 359-367, 2011.

MALBERGIER, A. et al. A paridade de gêneros e o uso de drogas: as meninas estão alcançando os meninos? Revista Brasileira de Psiquiatria, São Paulo, v. 34, n. 1, mar. 2012.

MALTA, D. C. et al. Prevalência do consumo de álcool e drogas entre adolescentes: análise dos dados da Pesquisa Nacional de Saúde Escolar. Revista Brasileira de Epidemiologia, São Paulo, v. 14, supl. 1, p. 136-146, 2011.
PRATTA, E. M. M.; SANTOS, M. A. Levantamento dos motivos e dos responsáveis pelo primeiro contato de adolescentes do ensino médio com substâncias psicoativas. Revista Eletrônica Saúde Mental Álcool Drogas, Ribeirão Preto, v. 2, n. 2, 2006.

RENNÓ JR., J. et al. Saúde mental da mulher no Brasil: desafios clínicos e perspectivas em pesquisa. Revista Brasileira de Psiquiatria, Rio de Janeiro, v. 27, supl. 2, p. S73-6, 2005.

RODRIGUES, J.; SANTOS, S. M. A.; SPRICCIGO, J. S. Ensino do cuidado de enfermagem em saúde mental na graduação em enfermagem. Acta paulista de enfermagem, São Paulo, v. 25, n. 6, p. 844-851, 2012.

VASCONCELOS, A. G. G. Perfil dos internos no sistema prisional do Rio de Janeiro: especificidades de gênero no processo de exclusão social. Ciência e Saúde Coletiva, Rio de Janeiro, v. 11, n. 2, p. 461-471, 2006.

\footnotetext{
Recebido para publicação em março de 2014

Versão final em fevereiro de 2015

Conflito de interesses: inexistente

Suporte financeiro: não houve
} 